was strongly associated with VTE risk assessment completion $(\mathrm{p}<0.05)$.

Conclusion. OA wards have hosted QI programmes with regards to VTE risk assessment which may be why VTE risk assessment was more likely to have been completed on OA wards. VTE risk assessment compliance overall is inadequate across all sites included in the study. Recommendations include further education for all ward staff on how, why and when VTE risk assessment should be completed, greater accessibility of an improved VTE risk assessment form and for QI initiatives on OA wards to be rolled out on WA wards. These findings have been presented and discussed at regional Trust teaching days, and this audit will be repeated in one year.

\section{An audit of liaison service provision in Aneurin Bevan University Health Board}

Jennifer Rankin* and Heledd Espley

Aneurin Bevan University Health Board

${ }^{*}$ Corresponding author.

doi: 10.1192/bjo.2021.297

Aims. Aneurin Bevan University Health Board (ABUHB) isundertaking a review of the Mental Health Liaison Service provision within it's acute general hospitals. The current liaison service is a small nurse led team which is available between $8 \mathrm{am}$ and midnight. ABUHB has recently opened a new Specialist Critical Care Hospital with the liaison service moving into a new base. A new model of care has been developed across the healthboard which has stretched the Liaison Service across several sites. Therefore, the liaison service may need to expanded to be able to provide high quality and timely care across a wide geographical area. The audit aims to idenitfy areas in which the liaison service is performing well in while identiying areas that need improvement. This audit may provide a focus for recommendations to enhance the current liaison provision.

Method. The liaison service was audited against RCPsych Psychiatric Liaison Accreditation Network (PLAN) quality standards. PLAN identified eighteen functions of a liaison team and provided details of quality standards within each function. These standards are either considered essential, expected or desirable. An accredited service is expected to meet $100 \%$ of essential standards, $80 \%$ of expected standards and $60 \%$ of desirable standards. Data were taken from a combination of sources including ABUHB policies, service managers and senior clinicians within both mental health and acute services.

Result. When comparing the current liaison service provision in ABUHB, $30 \%$ of essential standards were not met and $21 \%$ were only somewhat met. Particular domains that were identifed as needing improvement included policies and procedures and urgent and emergency mental health care. $36 \%$ of expected standards were met with $41 \%$ not met. Notable domains that the service was performing poorly in included governance; induction, and providing teaching and support to acute colleagues. $89 \%$ of desirable standards were not met.

Conclusion. The audit idenitifed that the current liaison service fails to meet core standards set out by RCPsych. This audit provides quantitative data to demonstrate that the liaison service is in need of improvement and investment. As a result, enhaving the current liaison service is now a priortity for the health board. A business case is being developed to consider enhancing the liaison service with a view to developing a Consultant led multidisciplinary team. The business case can use PLAN quality standards to make recommendations for improvements to the service.

\section{A clinical audit to investigate polypharmacy and interactions in inpatients in an old age psychiatric ward}

\author{
Mohan Rathnaiah ${ }^{1 *}$, Beth Brailsford ${ }^{2}$ and Nisha Mokashi ${ }^{3}$ \\ ${ }^{1}$ Derbyshire Healthcare NHS FT; ${ }^{2}$ UHDB NHS Founation Trust and \\ ${ }^{3}$ Nottinghamshire Healthcare NHS Foundation Trust \\ ${ }^{\star}$ Corresponding author.
}

doi: $10.1192 /$ bjo.2021.298

Aims. To identify any problematic polypharmacy in the patient records of those staying in Cherry Ward, an old age psychiatric unit at Highbury Hospital, Nottingham in the calendar year 2018. Background. Multi-morbidity is defined as more than one longterm medical condition in a single individual and is a factor that is closely associated with polypharmacy, the use of multiple medications concurrently. Appropriate and Problematic are the two classifications of polypharmacy outlined by the King's Fund report, the first describing optimised evidenced-based pharmacological management of comorbidities and the latter to label prescribed medications whose use is not in the best interests of the patient. The risk of drug interactions and adverse drug reactions is increased with polypharmacy, and frail elderly patients are particularly at risk of the side-effects of psychotropic medications used in the management of mental health disorders. Guidelines highlight this group as a key party to be identified when searching for at-risk people.

Method. The electronic records of those admitted and discharged from Cherry Ward in 2018 were reviewed in the period spanning January to May 2019, and the first forty-three patients were analysed in Microsoft Excel using criteria based on the King's fund report and the Medscape and BNF (British National Formulary) drug interaction tools. The Medscape drug interaction checker was used for initial screening; the complete medication list for each patient was entered into it and the number of interactions was displayed with advice on severity. If necessary, the individual interactions for each specific medication could also be crossreferenced in the BNF using the extensive lists provided for each drug. These are also graded from mild to severe.

Result. On discharge, $69.7 \%$ (thirty patients) met the criteria for being at higher risk of polypharmacy. One patient became at higher risk of polypharmacy during admission, and another two stepped down from meeting the criteria on admission but not on discharge. Thirty-one of the forty-three patients had at least one interaction recorded; $18.6 \%$ (eight patients) had at least one potentially severe interaction.

Conclusion. A substantial proportion of patients in Cherry ward in 2018 were at a higher risk of polypharmacy, reflecting current practice as outlined in the King's Fund report. Problematic polypharmacy is common among older patients hospitalised with psychiatric illness. Recommendations include use of an automated electronic system to investigate and flag up problematic polypharmacy and severe medication interactions.

\section{Cervical screening in assertive outreach team patients}

Hannah Reynolds*, Samaila Bello and Hanna Leech

Birmingham and Solihull Mental Health NHS Foundation Trust ${ }^{\star}$ Corresponding author.

doi: 10.1192/bjo.2021.299

Aims. To assess the uptake of cervical screening in patients under Birmingham and Solihull Assertive Outreach Teams; this 
included a re-audit of patients under the Central Birmingham Assertive Outreach Team.

Background. Patients with severe and enduring mental illness are known to have poorer physical health outcomes. In Birmingham and Solihull there are 6 Assertive Outreach Teams. These teams manage patients with a diagnosis of psychosis who have complex needs requiring intensive multidisciplinary input and often struggle to engage with health services. The national cervical screening programme aims to prevent cervical cancer by detecting and treating cervical abnormalities. Acceptable coverage is defined as screening at least $80 \%$ of people aged $25-49$ years within the last 3.5 years and $80 \%$ of people aged 50-64 years within the last 5.5 years. In $201871.4 \%$ of women in England and $70.9 \%$ in the West Midlands were screened adequately. An audit of 15 patients under the Central Birmingham Assertive Outreach Team in 2014 showed $46.2 \%$ had taken up screening, measured in the last 5 years for those aged 50-64 years and the last 3 years for those aged 25-49 years.

Method. A list was obtained of all female patients under the Assertive Outreach Teams with patients excluded if they were under 25 years or over 64 years or if they were known to have undergone a total hysterectomy. All GP practices with eligible patients registered to them were written to requesting the date of the patient's most recent smear test. Cervical screening was classed as in date if carried out in the last 3.5 years for patients aged 25-49 years or 5.5 years for patients aged 50-64 years.

Result. Out of 127 eligible patients, 110 had correct GP details on their record. Responses were received regarding 101 patients, 48 of whom had in date cervical screening (47.5\%). Of 58 patients aged 25-49 years, 26 had in date cervical screening (44.8\%). Of 43 patients aged 50-64 years, 22 had in date cervical screening (51.2\%). Conclusion. $13.4 \%$ patients did not have a known GP practice, increasing the risk of multiple poor physical health outcomes. The rates of cervical screening among Assertive Outreach Team patients are similar to the original audit in 2014 and fall significantly below the national standards and averages. These findings, along with the importance of working together to address the need for physical health monitoring in this population, will be communicated with the local Assertive Outreach Teams and GP practices.

\section{A closed loop audit of clerking psychiatric histories in an acute psychiatric inpatient unit}

\section{Samuel Richard Smith ${ }^{1 *}$, Rajiv Ark ${ }^{2}$}

and Thirunavukkarasu Aravinth ${ }^{3}$

${ }^{1}$ Lancaster University, Lancashire \& South Cumbria NHS Foundation Trust; ${ }^{2}$ Lancashire \& South Cumbria NHS Foundation Trust, Lancaster University and ${ }^{3}$ Lancashire \& South Cumbria NHS Foundation Trust

${ }^{*}$ Corresponding author.

doi: $10.1192 /$ bjo.2021.300

Aims. An accurate and complete history is a key component of a medical consultation. Evidence suggests that up to $80 \%$ of diagnosis may be made entirely off the patient history. The aim of this closed loop audit was to examine the effects of a clerking pro forma on the quality of doctors clerking histories of new patients admitted to an acute psychiatric inpatient unit, against standards suggested in the New Oxford Textbook of Psychiatry.

Method. Data for this audit were gathered by finding the initial clerking history for inpatients at The Orchard on ECR and RIO. The clerking histories of the 18 inpatients present on 12.10.20 were initially audited. These standards recommend in the in the New Oxford Textbook of Psychiatry include; Patient Identification (ID), Presenting Complaint (PC), History of Presenting Complaint (HPC), Psychiatric history, Medical history, Family history, Forensic history, Social history, Personal history, Premorbid personality, Mental state exam (MSE). After analysis of the results of the first loop, a clerking pro forma was created and distributed to junior doctors to implement. The clerking histories for the subsequent 18 patients to be admitted were then audited and compared.

Result. The results of the first audit cycle were poor. Only patient identification and presenting complaint were present in $100 \%$ of clerked histories. Concerningly, only $72 \%$ of the histories included the patients' medical histories, forensic histories were included $44 \%$ of the time, and social history just $39 \%$ of the time.

The implementation of a clerking history proforma showed improvements in all areas of clerking. Patient ID, PC, HPC, psychiatric history and MSE were now present in $100 \%$ of clerked histories. Forensic history showed a statistically significant improvement from $44 \%$ to $73 \%[\mathrm{X} 2(1)=5.9 ; \mathrm{p}=0.015]$. Social history showed a statistically significant improvement from $39 \%$ to $78 \%[\mathrm{X} 2(1)=5.6 ; \mathrm{p}=0.018]$. Premorbid personality showed a statistically significant improvement from $44 \%$ to $89 \%$ [X2(1) = $8.0 ; \mathrm{p}=0.005]$. Personal history showed a non-statistically significant improvement from $39 \%$ to $56 \%$, as did medical history from $72 \%$ to $94 \%$, and family history from $39 \%$ to $61 \%$.

Conclusion. In conclusion, the implementation of a clerking history pro-forma has significantly improved the quality and completeness of clerking histories gathered by doctors at The Orchard. This is hopefully increase diagnostic accuracy and improve the quality of care of patients in the hospital.

\section{Counting ECGs in acute psychiatry - The patients' price for junior doctors' rotations}

Marianna Rogowska*, Adam Montgomery and Luiz Dratcu

John Dickson Ward, Maudsley Hospital

${ }^{*}$ Corresponding author.

doi: 10.1192/bjo.2021.301

Aims. On 05/08/20, when a new cohort of doctors rotated onto an acute ward, (John Dickson Ward, Maudsley Hospital, London) a new handover tool on MS Teams was introduced, which replaced previously used MS Word document. The new handover tool can be accessed and edited by any of the users in the team. We hypothesised that the introduction of an interactive, live-updated tool would help improve physical health monitoring for patients, especially compliance with ECG taking. The aim of this project was to test this hypothesis.

Method. Authors have reviewed electronic documentation of patients admitted to and discharged from John Dickson Ward between $01 / 04 / 2020$ and 24/12/2020. Evidence of whether an ECG was performed, was offered but declined by the patient, or was not offered were noted in the final audit. Patients were divided into 3 groups: (1) Patients admitted and discharged from 01/04/20 - 05/08/2020; (2) Patients admitted and discharged from 05/08/2020 - 24/12/20, and (3) Patients admitted before the intervention date, but discharged after the date (i.e., the period when new junior doctors had rotated onto the ward). Fifty patient records were identified in Group 1, fifty in Group 2, and 18 in Group 3.

Result. Surprisingly, the percentage of patients who had a documented ECG did not improve after the intervention, with $37 / 50$ (74\%) of patients having an ECG in Group 1, and 37/50 (74\%) of patients having an ECG in Group 2. However, an incidental 